\title{
On the Wave Zone of Synchrotron Radiation
}

\author{
V.G. Bagrov * \\ Tomsk State University, 634050, Tomsk, Lenin Ave. 36, Russia, \\ Institute of High Current Electronics, SB RAS \\ 634055, Tomsk, Akademichesky Ave. 4, Russia
}

July 20, 2018

\begin{abstract}
The extension of the wave zone of synchrotron radiation is studied.
\end{abstract}

Keywords: wave zone, synchrotron radiation.

A theoretical study into the problem of the wave zone of synchrotron radiation was carried out in Ref. [1] (pp. 45-49). This research was published in English two years later [2] (pp. 3437 ). Since then, for half a century, these results (concerning the issue of wave radiation zone) have been considered as rigorously proven. Indeed, the proof given in Refs. [1, 2] remains valid; however, the possibility of applying these reasonings to an estimation of the wave zone size has to be refined. This problem is the subject of the present work.

The radiation power $W$ of a charged particle in classical electrodynamics is given by the expression

$$
W=\oint_{\mathbf{s}}(\mathbf{S} d \mathbf{s})
$$

where the surface $\mathbf{s}$ is a sphere with its center at the charge location, and the vector $\mathbf{S}$ is the Poynting vector

$$
\mathbf{S}=\frac{c}{4 \pi}[\mathbf{E H}]
$$

The electric $\mathbf{E}$ and magnetic $\mathbf{H}$ radiation fields of a point charge, determined with the help of Liénard-Wiechert potentials, can be written in the form

$$
\begin{gathered}
\mathbf{E}=\frac{e}{R^{2}[1-(\mathbf{n} \boldsymbol{\beta})]^{3}}\left\{\left(1-\beta^{2}\right)(\mathbf{n}-\boldsymbol{\beta})+\frac{R}{c}[\mathbf{n}[(\mathbf{n}-\boldsymbol{\beta}) \dot{\boldsymbol{\beta}}]]\right\}, \\
\mathbf{H}=[\mathbf{n E}], \quad \mathbf{n}=\frac{\mathbf{R}}{R} .
\end{gathered}
$$

Here, $\mathbf{v}=c \boldsymbol{\beta}$ is the charge velocity; $\mathbf{R}$ is the vector connecting the location point of the radiating charge to the observation point of radiation; $e$ is the charge magnitude; $c$ is the speed of light. In the right-hand sides of relations (3), all the quantities are regarded at the time instant $\tau$ determined by the condition

$$
\tau+\frac{R(\tau)}{c}=t
$$

*bagrov@phys.tsu.ru 


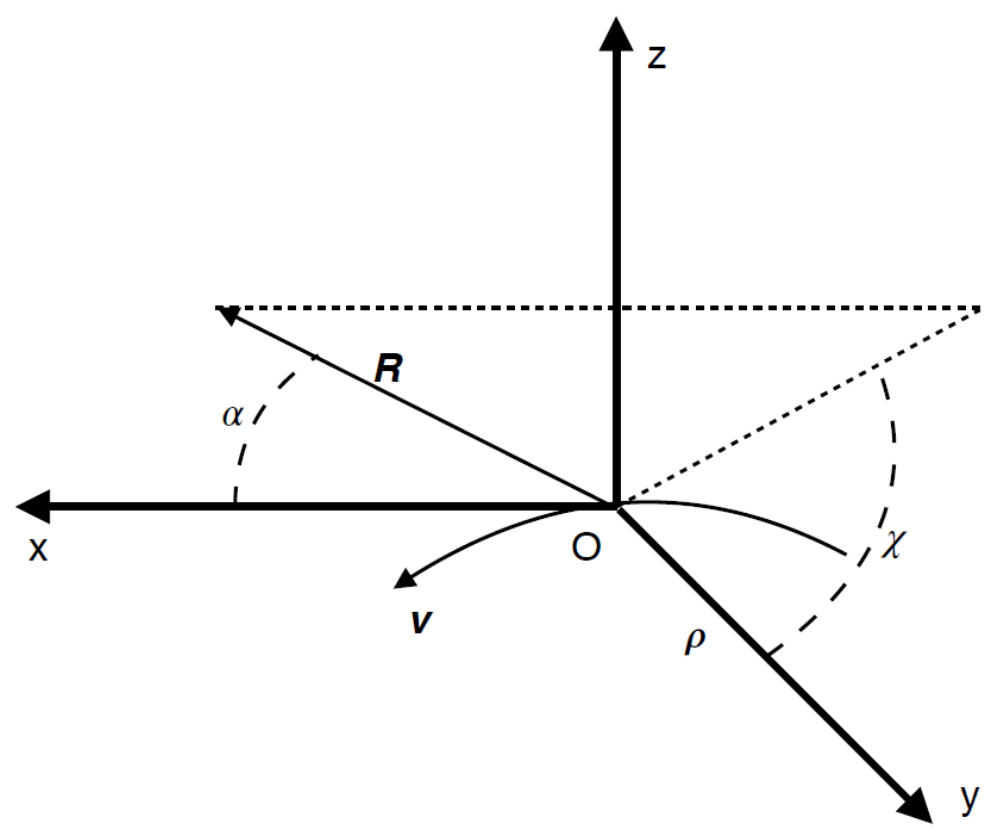

Figure 1: Coordinate system.

In particular, Eq. (44) implies

$$
\frac{\partial t}{\partial \tau}=1-(\mathbf{n} \boldsymbol{\beta})
$$

When considering synchrotron radiation, the velocity and acceleration of a particle are mutually orthogonal, constant in absolute value, and belong to one and the same fixed plane. Let us select the following coordinate system (see Fig. 1). The origin of the coordinate system is chosen at the location point of the radiating charge. The $x$-axis is directed along the velocity of the electron, and the $y$-axis is directed toward the center of the circular trajectory. We choose the $z$-axis so that the coordinate system is right-handed (when the charge moves in a constant and uniform magnetic field, with the $z$-axis being parallel to the external magnetic field). The radius of a circular orbit of the radiating particle is denoted by $\rho$ and is oriented along the $y$-axis of the given coordinate system. The angle formed by the $x$-axis and the vector $\mathbf{R}$ is denoted by $\alpha(0 \leqslant \alpha \leqslant \pi)$, while the angle between the $y$-axis and the projection of the vector $\mathbf{R}$ onto the $y z$-plane is denoted by $\chi(0 \leqslant \chi<2 \pi)$. The surface element is given by $d \mathbf{s}=\mathbf{R} R d \Omega, d \Omega=\sin \alpha d \alpha d \chi$.

Taking into account the above notation and Eqs. (11) and (2), one easily obtains a well-known expression $[1,2]$ for the instantaneous power of synchrotron radiation,

$$
W=\frac{c e^{2} \beta^{4}}{4 \pi \rho^{2}} \int_{0}^{\pi} \int_{0}^{2 \pi} \psi \sin \alpha d \alpha d \chi,
$$

where, in virtue of relations (4) and (5), the right- and left-hand sides of Eq. (6) are regarded at the radiation instant $\tau$, with the following notation being used:

$$
\begin{gathered}
\psi=a_{0}(\beta ; \alpha, \chi)+a_{1}(\beta ; \alpha, \chi) k+a_{2}(\beta ; \alpha, \chi) k^{2} \\
a_{0}=\frac{(\beta-\cos \alpha)^{2}+\left(1-\beta^{2}\right) \sin ^{2} \alpha \sin ^{2} \chi}{(1-\beta \cos \alpha)^{5}}
\end{gathered}
$$




$$
\begin{gathered}
a_{1}=\frac{2 \sin \alpha(\beta-\cos \alpha) \cos \chi}{(1-\beta \cos \alpha)^{5}}, \quad a_{2}=\frac{\sin ^{2} \alpha}{(1-\beta \cos \alpha)^{5}} ; \\
k=\frac{1-\beta^{2}}{\beta} \frac{\rho}{R} .
\end{gathered}
$$

The expression in Eq. (77) is a second-order polynomial in the parameter $k$ with three coefficients, $a_{s}(\beta ; \alpha, \chi)(s=0,1,2)$, depending on the value of $\beta$ and on the integration angles $\alpha, \chi$. For $R \rightarrow \infty$ (according to Eq. (8), this corresponds to the limit $k \rightarrow 0$ ), the given expression results in the instantaneous angular distribution $a_{0}(\beta ; \alpha, \chi)$ of synchrotron radiation power.

Formally, the value of $k$ in Eq. (7) is an expansion parameter, and therefore in Refs. [1, 2] we investigated the region $k<1$, with the tacit assumption that this is the radiation wave zone. However, the smallness of the parameter $k$ and the definition of the wave zone are related only indirectly: the parameter $k$ may be small, but the wave zone has not yet been formed.

Let us point out two obvious facts: a) the specific structure of the coefficients $a_{s}(\beta ; \alpha, \chi)$ may alter the conclusion about the boundaries of the wave radiation zone; however, this structure was not investigated in Refs. [1, 2]; b) if we consider radiation in a certain cone (the cone is given by the region of integration with respect to $\alpha, \chi)$, then the wave zone is determined not only by the radiation properties, but also by the choice of the cone in question. This fact was not considered in Refs. [1, 2], either.

Let us examine the total power of synchrotron radiation. Substituting the expressions (7) and (8) into Eq. (6), we carry out integration over $\alpha, \chi$ in the entire space. As a result of simple calculations, we find

$$
W=W_{0} \Phi_{0}, \quad W_{0}=\frac{2 c e^{2} \beta^{4}}{3 \rho^{2}\left(1-\beta^{2}\right)^{2}}, \quad \Phi_{0}=1+\bar{k}^{2}, \quad \bar{k}=\sqrt{\frac{1-\beta^{2}}{\beta^{2}}} \frac{\rho}{R} .
$$

Here, $W_{0}$ is the total emitted power in the limit $R \rightarrow \infty[1,2]$, and the coefficient $\bar{k}$ characterizes the size of the near-field zone.

We assume the space region $\bar{k}<1$ to be the radiation wave zone. From Eqs. (8) and (9), it then follows that $k$ and $\bar{k}$ are related by

$$
\gamma k=\bar{k}, \quad \gamma=\frac{1}{\sqrt{1-\beta^{2}}},
$$

where $\gamma$ is the relativistic factor (assuming, for instance, the electron energy to be $\sim 1 \mathrm{GeV}$, we obtain for the relativistic factor the approximation $\gamma \sim 2000$ ). Eq. (10) implies that $k<\bar{k}$, and also the fact that $\bar{k}<1$ is not necessarily the case at $k<1$. Consequently, the condition $k<1$, discussed in Refs. [1, 2, is only a necessary one, but is not sufficient to determine the synchrotron radiation wave zone $(k<\bar{k}<1)$.

From Eq. (9), it follows that

$$
\bar{k}=\frac{R_{v}}{R}, \quad R_{v}=\sqrt{\frac{1-\beta^{2}}{\beta^{2}}} \rho=\frac{\rho}{\sqrt{\gamma^{2}-1}},
$$

where $R_{v}$ is the distance separating the radiation space into the wave zone $\left(R_{v}<R\right)$ and the near-field zone $\left(R_{v}>R\right)$. The classical theory $[1,2]$ predicts a relation between the orbit radius $\rho$, the velocity of the radiating particle, and the strength $H$ of the controlling external magnetic field:

$$
\rho=\sqrt{\frac{\beta^{2}}{1-\beta^{2}}} \frac{m_{0} c^{2}}{|e H|}=\sqrt{\gamma^{2}-1} \frac{m_{0} c^{2}}{|e H|},
$$


where $m_{0}$ is the rest mass of the radiating particle. Substituting the expression (12) into Eq. (11), we obtain $R_{v}$ in the form

$$
R_{v}=\frac{m_{0} c^{2}}{|e H|}=\frac{c}{\omega_{c}}, \quad \omega_{c}=\frac{|e H|}{m_{0} c}
$$

where $\omega_{c}$ is cyclotron frequency. For instance, the field $H=1 T=10000$ results in $R_{v}=0,1704$ $\mathrm{cm}$. The expression (13) shows that the parameter $R_{v}$ is determined only by the rest energy of the radiating particle and by the magnitude of the external magnetic field. The other characteristics (for example, speed) of the radiating particle do not influence the quantity $R_{v}$.

Let us consider two examples when the radiation cone does not cover the entire space.

As a first example, we represent the whole space as the sum of two $( \pm)$ subspaces: the subspace $(-)$ is given by the region $y \geqslant 0$ (which corresponds to $\cos \chi \geqslant 0$, and the center of the orbit lies in this subspace); the subspace $(+)$ is given by the region $y<0$ (which corresponds to $\cos \chi<0$, and the center of the orbit does not lie in this subspace). It is easy to obtain the expressions

$$
W_{( \pm)}=W_{0} \Phi_{( \pm)}, \quad \Phi_{( \pm)}=\frac{1}{2}\left(1+\bar{k}^{2} \mp \frac{3 \beta}{8} \bar{k}\right), \quad \Phi_{0}=\Phi_{(+)}+\Phi_{(-)} .
$$

As expected, the particle characteristics (being its speed $\beta$ ) now also enter the expressions (14) for $\Phi_{( \pm)}$; however, the condition $\bar{k}<1$ determines the wave radiation zone in this case as well.

As a second example, we represent the entire space as the sum of two, (in) and (out), subspaces: the (in)-subspace corresponds to the radiation value $(\boldsymbol{\beta S})>0$ (which determines the range of variation $0<\alpha<\pi / 2$ ); the (out)-subspace corresponds to the radiation value $(\boldsymbol{\beta S})<0$ (which determines the range of variation $\pi / 2<\alpha<\pi$ ). Thus, the space is divided into the (in)-part, in which the projection of radiation pulse on the particle velocity is positive, and the (out)-part, in which the projection of radiation pulse on the particle velocity is negative.

For the (in)-subspace, it is easy to obtain expression

$$
\begin{gathered}
W_{(\mathrm{in})}=W_{0} \Phi_{(\mathrm{in})}, \quad \Phi_{(\mathrm{in})}=\Phi_{(\mathrm{in})}^{0}(\beta)\left[1+A_{(\mathrm{in})}(\beta) \bar{k}^{2}\right] \\
\Phi_{(\mathrm{in})}^{0}(\beta)=\frac{16+\beta\left(3+\beta^{2}\right)\left(7-3 \beta^{2}\right)}{32}, \quad A_{(\mathrm{in})}(\beta)=\frac{2\left(8-9 \beta+3 \beta^{2}\right)(1+\beta)}{16-11 \beta+6 \beta^{2}-3 \beta^{3}} .
\end{gathered}
$$

We note the simplest properties of the functions $\Phi_{(\mathrm{in})}^{0}(\beta)$ and $A_{(\mathrm{in})}(\beta)$ in the segment $0 \leqslant \beta \leqslant 1$. The function $\Phi_{(\mathrm{in})}^{0}(\beta)$ increases monotonously,

$$
\Phi_{(\mathrm{in})}^{0}(0)=\frac{1}{2} \leqslant \Phi_{(\mathrm{in})}^{0}(\beta) \leqslant 1=\Phi_{(\mathrm{in})}^{0}(1)
$$

and the function $A_{(\text {in) }}(\beta)$ at the ends of the segment $A_{(\text {in) }}(0)=A_{(\text {in) }}(1)=1$ has a unique maximum at the point

$$
\beta_{\max }=\frac{3-\sqrt{5}}{2} \approx 0,38197, \quad A_{(\mathrm{in})}\left(\beta_{\max }\right)=\frac{10(18 \sqrt{5}-5)}{319} \approx 1,2617 .
$$

Thus, the variation domain of the function $A_{(\mathrm{in})}(\beta)$ is bounded:

$$
1 \leqslant A_{\text {(in) }}(\beta) \leqslant \frac{10(18 \sqrt{5}-5)}{319} \approx 1,2617 .
$$


Returning to the expressions (15) and taking into account Eqs. (16)-(18), we get

$$
W_{(\text {in })}=\left\{\begin{array}{l}
\frac{1}{2} W_{0} \Phi_{0} \text { non-relativistic limit }(\beta \ll 1) ; \\
W_{0} \Phi_{0} \text { ultra-relativistic limit }(1 \ll \gamma)
\end{array}\right.
$$

From Eq. (19), we find that in the nonrelativistic case half of the emtted energy falls on the (in)-subspace, whereas in the ultra-relativistic case this subspace accumulates all the emitted energy. The emergence condition for the wave zone still has the form $\bar{k}<1$.

For the (out)-subspace, we obtain the expression

$$
\begin{gathered}
W_{(\text {out })}=W_{0} \Phi_{(\text {out })}, \quad \Phi_{(\text {out })}=\Phi_{(\text {out })}^{0}(\beta)\left[1+A_{(\text {out })}(\beta) k^{2}\right], \\
\Phi_{(\text {out })}^{0}(\beta)=\frac{16-\beta\left(3+\beta^{2}\right)\left(7-3 \beta^{2}\right)}{32}, \quad A_{(\text {out })}(\beta)=\frac{2\left(8+9 \beta+3 \beta^{2}\right)}{(1+\beta)\left(16+11 \beta+6 \beta^{2}+3 \beta^{3}\right)} .
\end{gathered}
$$

From Eqs. (15) and (20), it follows that $\Phi_{0}=\Phi_{(\text {in })}+\Phi_{(\text {out })}$, which corresponds to the equality of the total radiation energy to the sum of the two energies in the subspaces. This also means $1=\Phi_{\text {(in) }}^{0}(\beta)+\Phi_{\text {(out) }}^{0}(\beta)$, which indicates that the radiation energy in the wave zone is equal to the sum of the two energies in the wave zone of the subspaces. The functions $\Phi_{\text {(out) }}^{0}(\beta)$ and $A_{\text {(out) }}(\beta)$ in the segment $0 \leqslant \beta \leqslant 1$ are bounded and monotonously decreasing functions of $\beta$ :

$$
\frac{1}{2} \geqslant \Phi_{(\text {out })}^{0}(\beta) \geqslant 0,1 \geqslant A_{(\text {out })}(\beta) \geqslant \frac{5}{9}
$$

In the nonrelativistic limit $(\beta \ll 1)$, as implied by Eqs. (21) and (19), we find $W_{\text {(out) }}=$ $W_{(\text {in) }}=\frac{1}{2} W_{0} \Phi_{0}$ (in this case, with allowance made for Eq. (10), it has been taken into account that $k=\bar{k}$ ).

In the ultra-relativistic limit $(1 \ll \gamma)$ for $A_{\text {(out) }}(\beta \sim 1) \approx \frac{5}{9}$, with account taken of Eq. (20), we find

$$
\Phi_{(\text {out })}=\Phi_{\text {(out) }}^{0}(\beta \sim 1)\left(1+\frac{5}{9} k^{2}\right),
$$

which differs significantly from the expression (9) involving $k$. Eq. (22) involves $k$, and in this case the wave zone emerges earlier than in Eq. (91). However, as noted above, the limit $\Phi_{\text {(out) }}^{0}(\beta \sim 1) \rightarrow 0$ holds true, and, according to Eq. (20), this results in $\Phi_{(\text {out })}(\beta \sim 1) \rightarrow 0$. Consequently, the radiation value $W_{\text {(out) }}$ in the ultra-relativistic limit is extremely small in relation to $W_{(\mathrm{in})}$ and does not offer any tangible contribution to the total radiation.

For a given external magnetic field $H$ in the ultra-relativistic limit, the radiation value $W_{\text {(out) }}$ is not only small in relation to $W_{\text {(in) }}$, but also tends to zero as the energy of the radiating charge increases. Indeed, taking into account Eq. (9) for the quantity $W_{0}$ and using Eq. (12), we find the expression

$$
W_{0}=\frac{2 e^{2} \beta^{2} \omega_{c}^{2}}{3 c\left(1-\beta^{2}\right)}=\frac{2 e^{2} \omega_{c}^{2}}{3 c}\left(\gamma^{2}-1\right),
$$

which implies that the radiation value $W_{0}$ (and thereby also the value of $W_{\text {(in) }}$ ) for some fixed $H$ (with a fixed $\left.\omega_{c}\right)$ increases infinitely with an increasing charge energy $\left(\sim \gamma^{2}\right)$. For $W_{\text {(out) }}$ in Eq. (20), we get

$$
\begin{gathered}
W_{\text {(out) }}=\frac{e^{2} \omega_{c}^{2}}{c} \beta^{2}\left(1-\beta^{2}\right) B_{(\text {out })}(\beta)\left[1+A_{(\text {out })}(\beta) k^{2}\right], \\
B_{\text {(out) }}(\beta)=\frac{16+11 \beta+6 \beta^{2}+3 \beta^{3}}{48(1+\beta)^{2}} .
\end{gathered}
$$


The value of $B_{\text {(out) }}(\beta)$, decreasing monotonously in the segment $0 \leqslant \beta \leqslant 1$, is bounded: $\frac{1}{3} \geqslant B_{\text {(out) }}(\beta) \geqslant \frac{3}{16}$. From Eq. (24) , it follows that a fixed $H$ results in a radiation value $W_{\text {(out) }}$ decreasing to zero as the energy $\left(\sim \gamma^{-2}\right)$ increases.

It has been shown that the wave zone of synchrotron radiation is determined by the condition $\bar{k}<1$ (expressions (9) or (11)), which differs from the condition of Refs. 1, 2].

Acknowledgments. This work was supported in part by the RFBR (grant No. 18-02-00149) and by the Program for Improving TSUs Competitiveness among World Leading Scientific and Educational Centers.

\section{References}

[1] A. A. Sokolov, I. M. Ternov, V. G. Bagrov. The Classical Theory of the Synchrotron Radiation. In: The Synchrotron Radiation. Eds. A. A. Sokolov, I. M. Ternov. Moscow: Nauka, 1966. P. 18-71. (In Russian).

[2] A. A. Sokolov, I. M. Ternov. Synchrotron Radiation. Academie-Verlag, Berlin, 1968. P. 202. 